\title{
Holding on to a Cold Plasma
}

\author{
Researchers have demonstrated that an ultracold neutral plasma can be \\ magnetically confined, paving the way toward experiments that simulate \\ its hot astrophysical counterparts.
}

\section{By Sophia Chen}

( Itracold neutral plasmas (UCNPs) offer a controllable platform for studying phenomena not seen in their hotter cousins. But their lack of overall electrical charge makes neutral plasmas difficult to confine and manipulate. Now, Grant Gorman of Rice University, Texas, and colleagues have trapped a UCNP using magnetic fields [1]. Their technique could allow experiments that simulate the solar wind or aspects of white dwarf stars.

Physicists usually maintain plasmas in an ionized state using high temperatures, yielding plasmas whose dynamics are dominated by fast-paced thermal interactions. But this state can also be generated in gases close to absolute zero, producing plasmas in which positive ions move sluggishly and electron correlations play a significant role. Certain astrophysical plasmas are also in this strong-coupling regime, making UCNPs an important model system.

The researchers created a UCNP by laser cooling a gas of strontium atoms to $3 \mathrm{mK}$ and then stripping an electron from

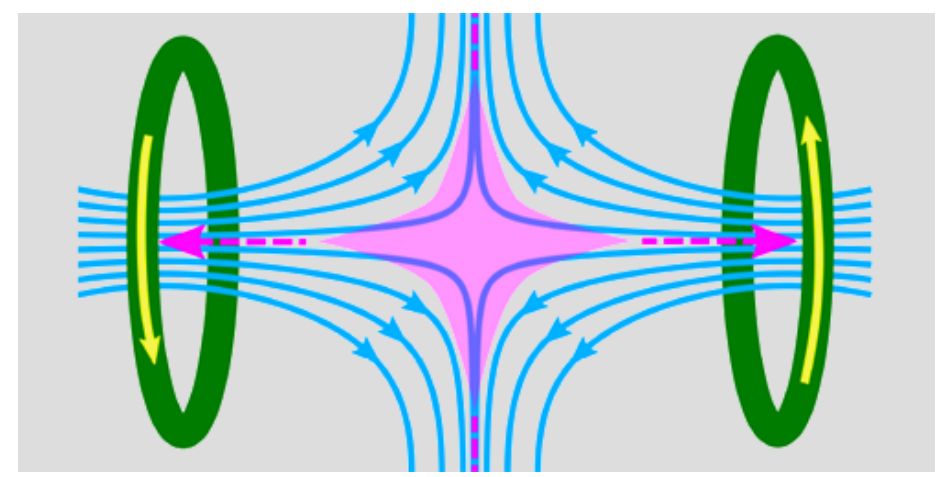

Credit: G. M. Gorman et al. [1] each atom using a light pulse. This process delivers very little energy to the electrons and ions, keeping the plasma ultracold and making it sensitive to relatively weak magnetic forces. The team applied such forces using a nonuniform magnetic field.

After being created at the center of the field, where the net field strength was zero, the plasma expanded rapidly. As the plasma moved into the stronger-field region, its expansion slowed. Gorman and colleagues didn't observe the plasma's ultimate escape from the trap, but they managed to contain it for at least half a millisecond. In the absence of magnetic confinement, such a plasma would dissipate in a few tens of microseconds. In future work, the researchers plan to combine magnetic fields with lasers to create even more robust and versatile traps.

Sophia Chen is a freelance science writer based in Columbus, Ohio.

\section{REFERENCES}

1. G. M. Gorman et al., "Magnetic confinement of an ultracold neutral plasma," Phys. Rev. Lett. 126, 085002 (2021). 